\title{
Using Patient Race, Ethnicity, and Language Data to Achieve Health Equity
}

\author{
Marshall H. Chin, MD, MPH \\ Richard Parrillo Family Professor of Healthcare Ethics, Department of Medicine, University of Chicago, Chicago, Illinois, USA.
}

$\mathrm{J}$ Gen Intern Med 30(6):703-5

DOI: $10.1007 / \mathrm{s} 11606-015-3245-2$

() Society of General Internal Medicine 2015

$\mathrm{C}$ ollecting data on patient race, ethnicity, and language (REL) is an important step in reducing health care disparities, as clinical performance measures can then be stratified by REL to guide quality improvement efforts. ${ }^{1}$ In this month's issue of JGIM, Klinger et al. report important inaccuracies in REL data in electronic health records (EHRs) in 13 primary care clinics. ${ }^{2}$ For example, $3 \%$ and $6.6 \%$ of patients who self-identified as Hispanic and African American, respectively, were not recorded as such in the EHR, and $20 \%$ of persons documented in the EHRs as English-speaking elected to take a survey in Spanish. Inaccuracies in REL data have clustered around the distinction between Latino and black, American Indians, and multiracial categories, and lack of granularity among Asian American and Latino subgroups. ${ }^{3}$ Klinger et al.'s study is the first to report inaccuracies in REL data in EHRs. Because meaningful use EHR regulations incentivize the collection of REL data, these data are more likely to be collected and available for electronic use. In this editorial, I will discuss how to accurately collect REL data, where REL data fit within comprehensive efforts to achieve health equity, the powerful incentives that are driving the collection of REL data, and emerging innovative uses of REL data.

The Health Research and Education Trust (HRET) has published best practices and training materials for REL data collection, ${ }^{4}$ and many of its recommendations are consistent with those of the Institute of Medicine. ${ }^{5}$ Patient self-reporting is the gold standard; thus, clerical staff must directly ask patients about their REL.

Patients generally understand the importance of collecting language data for improving communication and to determine

From the Section of General Internal Medicine, the Department of Medicine, the Chicago Center for Diabetes Translation Research, the MacLean Center for Clinical Medical Ethics, the Robert Wood Johnson Foundation (RWJF) Finding Answers: Disparities Research for Change National Program Office, and the RWJF Reducing Health Care Disparities Through Payment and Delivery System Reform Program Office, University of Chicago, Chicago, Illinois, USA.

Published online February 27, 2015 the need for interpreter services. The two key questions to ask are "How would you rate your ability to speak and understand English?" and "What language do you feel most comfortable speaking with your doctor or nurse?"4

However, patients sometimes question why race and ethnicity information are collected, ${ }^{3}$ and health care organizations must ensure that patients understand why REL data are important for improving the quality of care. For example, HRET recommends saying to patients, "Now I would like you to tell me your race and ethnic background. We use this information to review the treatment patients receive and make sure everyone gets the highest quality of care." "Do you consider yourself Hispanic/ Latino?" "Which category best describes your race?" HRET recommends following these two questions with a granular ethnicity screening question such as "I would like you to describe your race or ethnic background. You can use specific terms such as Korean, Mexican, Haitian, Somali." Even with this explanation of why REL data are collected, some patients remain suspicious. Thus, organizations must train their staff in how to respond to challenging patient questions, such as: "Do you discriminate against African-American patients like myself?", "Do you provide worse care for minority patients?", and "Are you trying to find out if I am a U.S. citizen?"

However, it is not enough to tell the intake clerical staff to ask patients REL questions as recommended above. Multiple departments must take action to collect and use REL data appropriately. The University of Chicago is currently working to improve the process of REL data collection, involving 15 different departments (e.g., information technology, patient care services, center for quality, managed care, population health, ambulatory, finance, etc.). Achieving accuracy in REL data collection is often an uphill battle unless leadership and staff of health care organizations make reducing disparities a priority.

The ultimate goal of REL data collection is improving the quality of care and outcomes for all patients and reducing health care disparities. Thus, REL data collection is not an end in itself. REL data alone are not a magic bullet that will end health care disparities, just as universal health insurance or cultural competency training for clinicians in isolation are not complete solutions for achieving equity. REL data are useful for stratifying clinical performance measures to identify disparities and thus increase 
an organization's motivation and ability to address these disparities. ${ }^{1}$ However, while over $90 \%$ of American Hospital Association member hospitals reported collecting REL data in 2013, only $23 \%$ declared that they used these data to identify disparities in clinical quality indicators. ${ }^{6}$ Moreover, stratified clinical performance data alone generally do not improve clinical outcomes such as hemoglobin A1c control in patients with diabetes. ${ }^{1}$ Health care organizations should stratify clinical performance data by REL and then implement culturally tailored quality improvement interventions.

While no magic bullet exists, each component of a comprehensive effort to reduce disparities plays an important role, including REL data collection and cultural competency training. For example, in 2013, the University of Chicago Medicine launched a diversity and inclusion initiative to transform the organization into one that is culturally and linguistically competent and that ensures equitable, high-quality care and optimal outcomes for all groups. The Medical Center Committee aims to eliminate variability in outcomes across population groups as measured by stratified performance metrics. Tailored quality improvement guided by stratified clinical performance data is an important part of the initiative, but the plan also includes recruitment, mentoring, advancement, and retention of diverse faculty and staff; community outreach; engagement of patients to improve their experience; and training of senior leadership and frontline staff in diversity issues. A comprehensive initiative that addresses the multiple drivers of health disparities is more likely to succeed. The Dean of the Biological Sciences Division and senior hospital leadership have made diversity and equity a top priority, both because it is the right thing to do, and because it is a good business strategy.

One common mistake made by health care organizations is delaying the disparity intervention process until clinical performance measures can be stratified by REL, which can take months to years. ${ }^{7}$ Several steps should be concomitant with improving REL data collection - and, in fact, facilitate this process - such as creating a culture of equity and getting buy-in for reducing disparities across all levels of the organization. ${ }^{1}$ In addition, while organizations are developing the capability to accurately collect REL data, they can begin quality improvement interventions to reduce disparities. Organizations can ask their providers, staff, and patients to suggest areas that are the most important qualitatively to work on, pick low-hanging fruit, and work with enthusiastic early adopters. They can also train staff in cultural competency, disparities, and quality improvement.

There are powerful arguments and incentives for the accurate collection of REL data. Reducing health care disparities is a moral and professional imperative, and stratified clinical performance data can motivate quality improvement. Moreover, various financial and non- financial incentives are either in existence now or on the horizon. REL data collection is already part of EHR meaningful use participation requirements for increasing reimbursement. The National Quality Forum has recommended that stratified performance measures be considered as part of routine public performance reporting by the Centers for Medicare \& Medicaid Services. ${ }^{8}$ Stratified measures are also helpful in pay-for-performance (P4P) incentive programs to help organizations identify populations with the worst outcomes whose improvement would most powerfully increase aggregate population levels of performance. In addition, it is likely that $\mathrm{P} 4 \mathrm{P}$ programs for reducing disparities will be implemented by payers over time. Stratified performance measures may also help non-profit hospitals demonstrate community benefit in order to maintain their non-profit tax status. Furthermore, the legality of REL data collection is firm. ${ }^{9}$ The regulations of Title VI of the 1964 Civil Rights Act prohibit discrimination based on race, and encourage preventive self-analysis to further this goal. Thus, evaluating and improving the quality of care by race and ethnicity help to demonstrate compliance with Title VI.

Incentives for population health management and efficient value-driven care are increasing with the proliferation of global payments, bundled payments, and accountable care organizations. In this environment, REL data collection could help organizations geo-map or "hot-spot" geographic areas with a high density of morbidity, and could facilitate addressing social determinants of health in conjunction with community-based organizations, public health organizations, and local governments. REL data can help identify areas for investment and resource allocation. For example, if cervical cancer screening rates were low in a community with many Vietnamesespeaking patients, REL data could help target patients needing language services and culturally tailored patient education materials and support.

In an era of Big Data, personalized medicine, and valuedriven care, REL and other sociodemographic data could be useful for predictive analytics to identify high-risk patients who might benefit from more intensive care and monitoring. ${ }^{10}$ While precautions must be taken to prevent misuse of such techniques, it is likely that predictive analytics will be employed with more sophistication over time, as their use in other industries for marketing and social networking have far outpaced that in health care.

We need to move REL data collection into the twentyfirst century. We have to catch up in implementing proven methods for the accurate collection of REL data, and go beyond. ${ }^{4}$ We must view REL data collection as one step in a comprehensive roadmap for reducing disparities, ${ }^{1}$ and a building block for evolving analytical tools to improve health outcomes. ${ }^{10}$ Today's opportunities for reducing health disparities are great. We must seize the moment, 
and tailor our solutions for different populations to achieve health equity.

Acknowledgments: Dr. Chin was supported by a National Institute of Diabetes and Digestive and Kidney Diseases Midcareer Investigator Award in Patient-Oriented Research (K24 DK071933) and the Chicago Center for Diabetes Translation Research (P30 DK092949).

Corresponding Author: Marshall H. Chin, MD, MPH; Richard Parrillo Family Professorship in Healthcare Ethics, Department of Medicine, University of Chicago, 5841 South Maryland Avenue, MC 2007, Chicago, Illinois 60637, USA (e-mail: mchin@medicine.bsd.uchicago.edu).

\section{REFERENCES}

1. Chin MH, Clarke AR, Nocon RS, Casey AA, Goddu AP, Keesecker NM, Cook SC. A roadmap and best practices for organizations to reduce racial and ethnic disparities in health care. J Gen Intern Med. 2012;27:9921000.

2. Klinger EV, Carlini SV, Gonzalez I, St. Hubert S, Linder JA, Rigotti NA, Kontos EZ, Park ER, Marinacci LX, Haas JS. Accuracy of race, ethnicity, and language preference in an electronic health record. $\mathrm{J}$ Gen Intern Med. 2015. doi:10.1007/s11606-014-3102-8.
3. Hasnain-Wynia R, Baker DW. Obtaining data on patient race, ethnicity, and primary language in health care organizations: current challenges and proposed solutions. Health Serv Res. 2006;41(4, part1):1501-1518.

4. Hasnain-Wynia R, Pierce D, Haque A, Hedges Greising C, Prince V, Reiter J. Health Research and Educational Trust Disparities Toolkit. 2007. Available at: http://www.hretdisparities.org (accessed January 28, 2015).

5. Ulmer C, McFadden B, Nerenz DR, eds. Race, Ethnicity, and Language Data: Standardization for Health Care Quality Improvement. Washington, DC: The National Academies Press; 2009.

6. Institute for Diversity in Health Management and Health Research and Educational Trust. Diversity and Disparities: A Benchmark Study of U.S. Hospitals in 2013. Available at: http://www.diversityconnection.org/ diversityconnection/leadership-conferences/diversity disparities_Benchmark_study_hospitals_2013.pdf (accessed December 25, 2014).

7. Cook SC, Goddu AP, Clarke AR, Nocon RS, McCullough KW, Chin MH. Lessons for reducing disparities in regional quality improvement efforts. Am J Manag Care. 2012;18(6 Suppl):s102-s105.

8. National Quality Forum. Risk adjustment for socioeconomic status or other sociodemographic factors: technical report. August 15, 2014 Available at: http://www.qualityforum.org/Publications/2014/08/Risk Adjustment_for_Socioeconomic_Status_or_Other_Sociodemographic_Factors.aspx (accessed February 6, 2015)

9. Rosenbaum S, Burke T, Nath Sw, Santos J, Thomas D. The legality of collecting and disclosing patient race and ethnicity data (legal barriers to health information law). Washington, D.C.: George Washington University, School of Public Health and Health Services, Department of Health Policy, 2006. Available at: http://hsrc.himmelfarb.gwu.edu/sphhs_policy_briefs/ 33/ (accessed December 28, 2014).

10. Bates DW, Saria S, Ohno-Machado L, Shah A, Escobar G. Big data in health care: using analytics to identify and manage high-risk and high-cost patients. Health Aff. 2014;33:1123-1131. 